\title{
Time-to-use of Intravenous Antibiotics in Patients with Sepsis in whom Activation of the Sepsis Fast Track Protocol was Facilitated by the National Early Warning Score
}

\author{
(1) Pannika Thuakta ${ }^{1}$, (1) Borwon Wittayachamnankul1 , (1) Titisuda Chounjai², (1) Theerapon Tangsuwanaruk ${ }^{1}$ \\ ${ }^{1}$ Department of Emergency Medicine, Faculty of Medicine, Chiang Mai University, Chiang Mai, Thailand \\ 2Maharaj Nakorn Chiang Mai Hospital, Chiang Mai, Thailand
}

\begin{abstract}
Aim: To examine changes in door-to-antibiotic time in pre- and post-intervention groups.

Materials and Methods: A quasi-experimental study was conducted in the Emergency Department involving adult patients who were diagnosed with sepsis or septic shock in a university-based hospital. The patients were distributed into one of two groups: a pre-intervention or post-intervention group. In the post-intervention group, among patients with a suspected infection and a National Early Warning Score (NEWS) of $\geq 5$, the sepsis fast track protocol was used in the normotensive group and the sepsis with shock fast track protocol was used in the hypotensive group. Our primary outcome was the difference in the door-to-antibiotic time in the pre- and post-intervention groups.

Results: Overall, 117 patients were included in the pre-intervention group and 102 patients in the post-intervention group. The median door-to-antibiotic time in the pre-intervention group was $45 \mathrm{~min}$ [interquartile range (IQR): 30-65], and the median door-to-antibiotic time in the post-intervention group was $30 \mathrm{~min}$ (IQR: 20-55, $p=0.009)$. However, there was no significant difference in the mortality rate ( $p=0.194)$.

Conclusion: Using an activated system with NEWS for screening patients suspected with sepsis helped reduce the door-to-antibiotic time.

Keywords: Antibiotic, emergency department, National Early Warning Score, protocol, sepsis
\end{abstract}

\section{Introduction}

Sepsis is associated with high mortality and morbidity (1). In 2017 the World Health Organization reported more than 30 million cases of sepsis worldwide, and more than six million deaths per year had been attributed to sepsis (2). The mortality rate reached $35.09 \%$, making sepsis the fourth leading cause of death worldwide $(3,4)$. To improve survival in cases of sepsis the Surviving Sepsis Campaign Bundle 2018, guidelines recommend administering empirical intravenous antibiotics within one hour and adequate fluid resuscitation in sepsis cases with hypotension or with serum lactate levels $\geq 4$ millimoles per liter ( $\mathrm{mmol} / \mathrm{L})(1,5$ 7).

An important factor in prompt management has been the introduction of an accurate and rapid detection tool (8). In a recent study it was found that the quick Sequential Organ Failure Assessment (qSOFA) predicted hospital mortality rates and intensive care unit (ICU) length of stay more accurately than the Systemic Inflammatory Response Syndrome (SIRS) (9). However very recent studies have found the National Early Warning Score (NEWS) to be as effective in the prediction of hospital mortality rate and ICU admission as QSOFA and more accurate in comparison to SIRS (9-12). Somehow, NEWS has been found to have more accuracy in predicting mortality and ICU admission when compared to qSOFA (12-14). However, no previous studies have reported the use of NEWS for early activation of the sepsis code to lessen the time to goal of therapeutic intervention including the administration of empirical antibiotics, fluid resuscitation, or to reduce mortality in the emergency department (ED). 
This study aimed to compare door-to-antibiotic time before and after implementation of NEWS as a sepsis screening tool, where a NEWS $\geq 5$ and suspected infection were used to trigger the activation of the sepsis fast track protocol.

\section{Materials and Methods}

\section{Study Design}

This study was a retrospective and prospective, quasiexperimental study which was approved by the Research Ethics Committee of Faculty of Medicine of Chiang Mai University (no: 377/2018, date: 03.10.2018). Written informed consent was obtained from patients who were enrolled on the prospective study. This study was registered in the Thai Clinical Trials Registry (www.clinicaltrials.in.th, TCTR20191002001).

\section{Study Setting and Population}

Patients who visited the ED from June 1, 2018 to August 31, 2018, prior to the implementation of NEWS as a sepsis trigger tool, were recruited onto the study. The qSOFA criteria had been used for the screening of these patients who were suspected sepsis and action had been taken as for a general patient without the sepsis fast track protocol. These patients were classified as the pre-intervention group. ED patients from October 1, 2018 to December 31, 2018, after the implementation of NEWS as a sepsis screening tool, were included in the post-intervention group. The inclusion criteria were as follows: patients aged 18 years or over with a suspected infection from a medical condition with NEWS $\geq 5$. The exclusion criteria included patients referred from another hospital without available medical records, patients requiring emergency surgery, patients discharged from the ED, patients with a misdiagnosis, patients who had received antibiotics within the last 30 days, or refusal by patient and/or guardian refusal or were not able to consent to participation in the research.

Data collection occurred at two time points: three months preintervention and three months post-intervention, intervention being the implementation of the sepsis fast track protocol. Patients in the pre-intervention group were recruited from June 1, 2018 to August 31, 2018, and included those diagnosed with sepsis or septic shock, according to the $10^{\text {th }}$ revision of the International Statistical Classification of Diseases and Related Health Problems, with a NEWS $\geq 5$. From September 1 to September 30, 2018, triage and screening personnel were briefed on the use of NEWS as a screening tool in patients with suspected sepsis (run in phase). If NEWS $\geq 5$, screening personnel were to alert the emergency physician, who would be the one to decide on the activation of the sepsis fast track protocol. The protocol was divided into two sections: a normotensive and a hypotensive group. The normotensive group included patients with a systolic blood pressure (SBP) $\geq 90$ millimeters of mercury $(\mathrm{mm} \mathrm{Hg}$ ) or a mean arterial pressure (MAP) $\geq 65 \mathrm{~mm} \mathrm{Hg}$, and appropriate antibiotics were given during treatment. The hypotensive group included patients with SBP $<90 \mathrm{~mm} \mathrm{Hg}$ or MAP $<65 \mathrm{~mm} \mathrm{Hg}$. In this group, in addition to appropriate antibiotics, adequate fluid resuscitation would be administered. Data for analysis of the postintervention group was collected from October 1 to December 1 , 2018 by both nurses and physicians. The emergency physicians and the nurses are the same team, no increase population, no have any benefit or punish in pre- and post-intervention group.

\section{Outcome Measures}

The primary outcome was the door-to-antibiotic time in patients when NEWS was used as a trigger tool to activate sepsis fast track protocol, in comparison to the door-to-antibiotic time in patients where such a tool was not used. The secondary outcomes examined the door-to-intravenous bolus time (door-to-IV bolus time), door-to-laboratory time (door-to-lab time) taken, lactate clearance, time to admission decision, average hospital length of stay (LOS), and average number of days ICU free, average ventilator free days, and 28-day mortality rate. The door-to-IV bolus time was defined as time between patient arriving at ED to IV bolus being achieved. The door-to-lab time taken was defined as time between patient arriving at ED to blood test taken. The lactate clearance was defined as percentage of blood lactate at arrival minus blood lactate concentration at follow up 2 hours after resuscitation. For lactate clearance, patients in whom there was no follow up of blood lactate, or blood lactate at visit $<2$ $\mathrm{mmol} / \mathrm{L}$ or missed data were not included in this outcome. The time to admission decision was defined as time between patient arriving at ED to the nurse receiving the admission order.

\section{Sample Size Calculation}

To ensure the number of patients in this study was adequate, the sample size was calculated by independent mean (15). A previous study had reported a mean of door to antibiotics time in the preintervention group as 139 minutes [standard deviation (SD)=74] and in the post-intervention group as 81 minutes $(S D=39)$ with an alpha error of 0.05 and a beta error of 0.1 , required a sample size of 22 patients in each group (8).

\section{Statistical Analysis}

Descriptive statistics were used for categorical data and mean values, standard deviation, medians, and interquartile ranges for continuous data. The Shapiro-Wilk test was used to test a normal distribution. Variables that approximated to a normal distribution were summarized as mean \pm SD, and groups were compared using t-tests. Other continuous or ordinary scaled variables were summarized as median, interquartile range, and groups and compared using Mann-Whitney U tests. All statistical 
analyses were performed using SPSS ${ }^{\circledR}$ Statistics version $22\left(\right.$ IBM $^{\circledR}$, Armonk, New York, USA). Statistical significance was designated as $p<0.05$ unless stated otherwise.

\section{Results}

There was a total of 539 patients over 18 years of age with sepsis or septic shock, with a NEWS $\geq 5$ and following application of the exclusion criteria, 275 patients were eligible for inclusion in this study. One hundred and seventeen patients were included in the pre-intervention group and 102 in the post-intervention group as shown in Figure 1. Baseline characteristics, including underlying diseases, hospital readmission within three months, vital signs, NEWS, lymphocyte count, and serum lactate did not significantly differ between the pre-and post-intervention groups (Table 1).

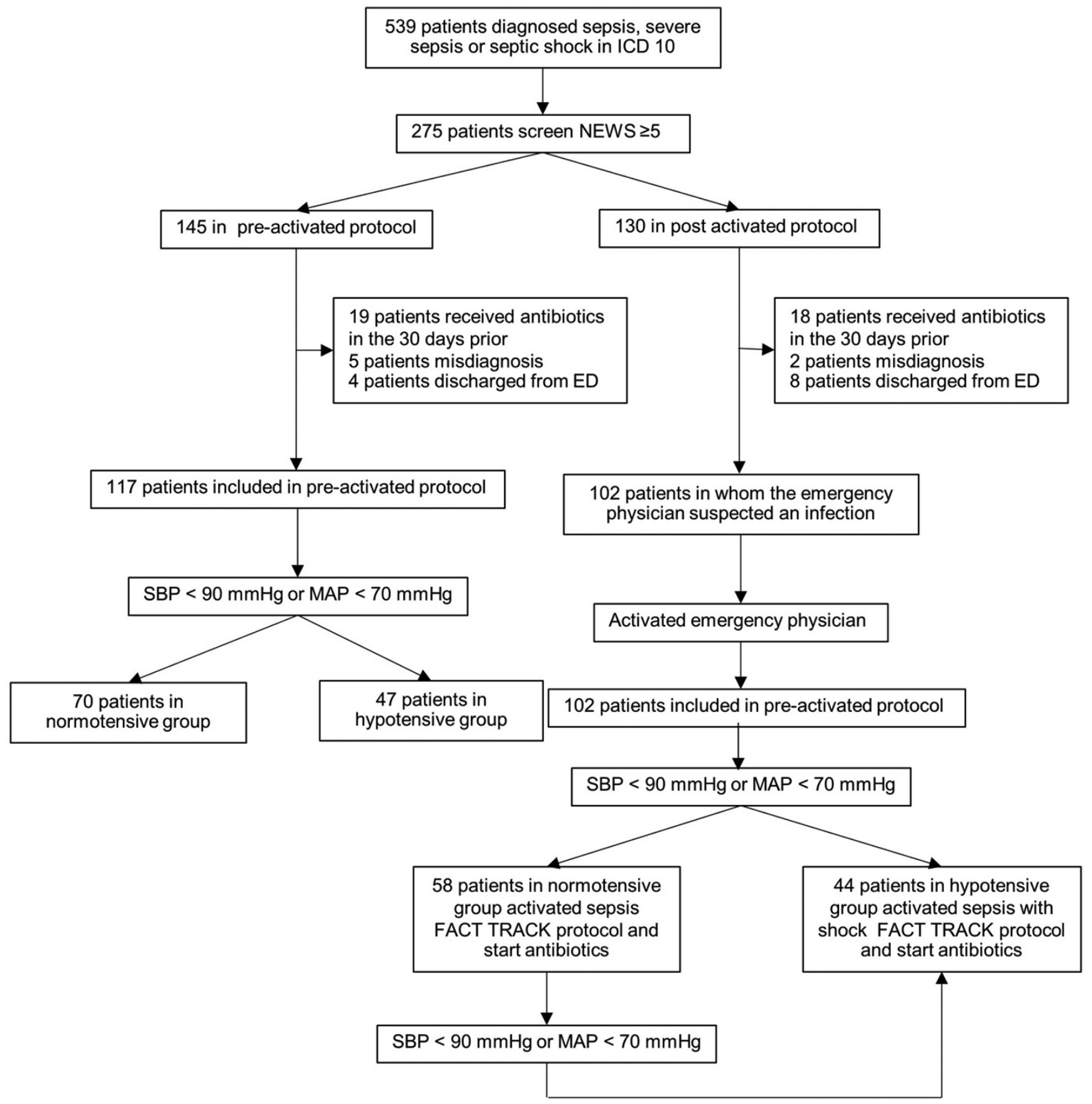

Figure 1. Study flow chart

ED: Emergency department, ICD 10: $10^{\text {th }}$ revision of the International Statistical Classification of Diseases and Related Health Problems, MAP: Mean arterial pressure, mmHg: Millimeters of mercury, NEWS: National Early Warning Score, SBP: Systolic blood pressure 


\begin{tabular}{|c|c|c|c|}
\hline & Pre-intervention $(n=117)$ & Post-intervention $(n=102)$ & $p$ value \\
\hline \multicolumn{4}{|l|}{ Group - n (\%) } \\
\hline Normotensive group & $47(42.0)$ & $44(43.14)$ & 0.657 \\
\hline Hypotensive group & $70(59.8)$ & $58(56.9)$ & 0.657 \\
\hline Male - n (\%) & $66(56.4)$ & $48(47.1)$ & 0.213 \\
\hline Age - years & $64.4 \pm 18.7$ & $65.5 \pm 18.2$ & 0.643 \\
\hline \multicolumn{4}{|l|}{ Medical condition - n (\%) } \\
\hline Diabetes mellitus & $19(16.2)$ & $22(21.6)$ & 0.404 \\
\hline Hypertension & $42(35.9)$ & $45(44.1)$ & 0.271 \\
\hline Dyslipidemia & $23(19.7)$ & $26(25.5)$ & 0.384 \\
\hline Cancer & $33(25.2)$ & $30(29.4)$ & 0.962 \\
\hline Chronic kidney disease & $17(14.5)$ & $18(17.6)$ & 0.658 \\
\hline Cerebrovascular disease & $17(14.5)$ & $24(23.5)$ & 0.126 \\
\hline Readmission within 3 months-n (\%) & $39(33.3)$ & $32(31.4)$ & 0.869 \\
\hline Temperature - degrees Celsius & $37.9 \pm 1.5$ & $38.0 \pm 1.3$ & 0.579 \\
\hline Heart rate - beats per min & $107.1 \pm 24.2$ & $111.5 \pm 25.9$ & 0.197 \\
\hline Respiratory rate - times per min & $29 \pm 16.9$ & $25 \pm 8.9$ & 0.102 \\
\hline SBP - mmHg (IQR) & $105.5(83.0-130.5)$ & $91.5(77.5-131.0)$ & 0.248 \\
\hline MAP - mmHg (IQR) & $72.5(61.0-90.5)$ & $67.5(58.0-100.5)$ & 0.072 \\
\hline Oxygen saturation - \% (IQR) & $90.0(84.0-96.0)$ & $93.5(89.0-97.0)$ & 0.693 \\
\hline NEWS - score (IQR) & $9.0(7.0-10.5)$ & $8.5(7.0-11.0)$ & 0.679 \\
\hline White blood cell count - x103 cell/cu.mm (IQR) & $12.0(5.6-21.2)$ & $11.6(6.3-16.0)$ & 0.843 \\
\hline Serum lactate - mmol/L (IQR) & $3.05(1.8-4.6)$ & $2.5(1.9-3.8)$ & 0.297 \\
\hline
\end{tabular}

After implementing the sepsis fast track protocol, the median door-to-antibiotic time was 34 minutes (20 to 55 ); this was statistically significantly lower from the pre-intervention median time of 45 minutes ( 30 to $65 ; p=0.009$ ). Median door-to-lab time taken in the post-intervention group was lower than the preintervention group [18 (8-40) vs $11(5-20) ; p=0.003]$. However, there were no difference in door-to-IV bolus time, time to an admission decision, average hospital LOS, average ICU free days, average ventilator free days, and 28-day mortality rate (Table 2).

Hypotensive patients and Normotensive patients were separated in a subgroup analysis, baseline characteristics of patients (Tables 3 and 4), including treatment received, did not significantly differ, apart from one exception, in the normotensive group there was a lower prevalence of cerebrovascular disease in the pre-group than in the post-group ( 12.9 vs $39.8 ; p=0.013$ ). In the post-intervention normotensive group, door-to-antibiotic time was found to be statistically significantly reduced, median pre-intervention 44.5 (30.0 to 64.3) minutes and post-intervention 25.0 (20.5 to 51.5) minutes; $p=0.003$, but there was no significant difference in the hypotensive group. Likewise, in the post-intervention group, door-to-lab time taken was also found to be reduced, again with statistical significance, median pre-intervention 16.5 (6.8 to 27.3) minutes, post-intervention 11.5 (5.5 to 20.0) minutes; $p=0.005$. There were no other significant differences found. With regards to the hypotensive group, time to admission decision increased, with statistical significance, median pre-intervention being 213.0 (173.0 to 300.0) minutes, and post-intervention 273.0 (196.3 to 327.5) minutes; $p=0.025$.

\section{Discussion}

This study found activation of code sepsis using NEWS to detect sepsis patients earlier significantly reduced time to antibiotic administration. Several studies have been conducted to compare different sepsis screening tools. Usman et al. (16) and Thodphetch et al. (17) found that NEWS as a sepsis screening tool had higher sensitivity in comparison to qSOFA, SIRS, and Search Out Severity Score. Screening tools with a higher sensitivity are believed to result in more rapid detection of sepsis and thus more prompt management. Seymour et al. (6) found that the administration of antibiotics prior to 0.95 hours can reduce the mortality rate 


\begin{tabular}{|l|l|l|l|}
\hline \multicolumn{2}{|l|}{ Table 2. Primary and secondary outcomes } \\
\hline & Pre-intervention (n=117) & Post-intervention (n=102) & $\mathbf{p}$ value \\
\hline Door-to-antibiotic time - min (IQR) & $45.0(30.0-65.0)$ & $30.0(20.0-55.0)$ & 0.009 \\
\hline Door-to-IV bolus time - min (IQR) & $12.0(6.0-32.0)$ & $8.5(5.0-20.0)$ & $11.0(5.0-20.0)$ \\
\hline Door-to-lab time taken - min (IQR) & $18.0(8.0-40.0)$ & $17.0 \pm 37.3$ & 0.150 \\
\hline Lactate clearance - \% & $23.1 \pm 28.7$ & $223.0(180.5-297.0)$ & 0.339 \\
\hline Time to admission decision - min (IQR) & $225.0(175.0-297.0)$ & $7.0(5.0-13.0)$ & 0.919 \\
\hline Average hospital LOS - day (IQR) & $8.0(4.0-17.0)$ & $7.0(3.0-10.0)$ \\
\hline Average ICU free days - day (IQR) & $6.0(3.0-12.0)$ & $7.0(3.0-10.0)$ \\
\hline Average ventilator free days - day (IQR) & $6.0(2.5-10.0)$ & $31(30.4)$ & 0.603 \\
\hline 28-day mortality rate-n (\%) & $35(29.9)$ & 0.885 \\
\hline $\begin{array}{l}\text { Data presented as mean } \pm \text { SD or median (interquartile range), depending on distribution. } \\
\text { ICU: Intensive care unit, IQR: Interquartile range, IV: Intravenous, lab: Laboratory, LOS: Length of stay, min: Minute, SD: Standard deviation, n: Number }\end{array}$ \\
\hline
\end{tabular}

\begin{tabular}{|c|c|c|c|c|c|c|}
\hline & $\begin{array}{l}\text { Pre-intervention } \\
\text { normotensive } \\
(\mathrm{n}=70)\end{array}$ & $\begin{array}{l}\text { Post-intervention } \\
\text { normotensive } \\
(\mathrm{n}=58)\end{array}$ & $\begin{array}{l}p \\
\text { value }\end{array}$ & $\begin{array}{l}\text { Pre-intervention } \\
\text { hypotensive } \\
(\mathrm{n}=47)\end{array}$ & $\begin{array}{l}\text { Post-intervention } \\
\text { hypotensive } \\
(\mathrm{n}=44)\end{array}$ & $\begin{array}{l}p \\
\text { value }\end{array}$ \\
\hline Male sex - n (\%) & $42(60.0)$ & $31(53.5)$ & 0.571 & $23(48.9)$ & $17(38.6)$ & 0.437 \\
\hline Age - year & $63.0 \pm 17.7$ & $67.3 \pm 17.8$ & 0.181 & $71.0(54.0-89.0)$ & $66.0(59.8-78.8)$ & 0.950 \\
\hline \multicolumn{7}{|l|}{ Medical condition - $\mathbf{n}(\%)$} \\
\hline Hypertension & $22(31.4)$ & $28(48.8)$ & 0.078 & $20(42.6)$ & $17(38.6)$ & 0.868 \\
\hline Dyslipidemia & $14(20.0)$ & $16(27.6)$ & 0.424 & $9(19.2)$ & $10(22.7)$ & 0.872 \\
\hline Cancer & $19(27.1)$ & $13(22.4)$ & 0.682 & $14(29.8)$ & $17(38.6)$ & 0.504 \\
\hline CKD & $13(18.6)$ & $14(24.1)$ & 0.582 & $4(8.5)$ & $4(9.1)$ & 1.000 \\
\hline Heart rate - beats per min & $108.7 \pm 19.7$ & $113.6 \pm 27.3$ & 0.235 & $104.8 \pm 29.9$ & $109.5 \pm 25.2$ & 0.417 \\
\hline Respiratory rate - times per min (IQR) & $29.0(24.0-37.0)$ & $28(22.5-35.0)$ & 0.218 & $28.0(22.0-40.0)$ & $24.0(20.5-32.0)$ & 0.583 \\
\hline $\mathrm{SBP}-\mathrm{mmHg}$ & $129.1 \pm 28.9$ & $138.9 \pm 28.5$ & 0.184 & $81.0(70.0-91.0)$ & $81.0(72.3-95.5)$ & 0.381 \\
\hline MAP - mmHg & $88.0(78.5-97.8)$ & $93.0(112.0-141.8)$ & 0.004 & $61.0(56.0-69.0)$ & $59.5(56.3-76.0)$ & 0.862 \\
\hline Oxygen saturation - \% (IQR) & $91.0(87.3-94.0)$ & $92.0(89.3-95.0)$ & 0.530 & $88.0(78.0-97.0)$ & $95.0(86.8-98.0)$ & 0.199 \\
\hline NEWS - score (IQR) & $8.0(7.0-9.0)$ & $8.0(7.0-10.0)$ & 0.325 & $9.5 \pm 3.3$ & $9.0 \pm 2.8$ & 0.416 \\
\hline White blood cell count $\times 10^{3}$ cell/cu.mm (IQR) & $13.4(4.5-20.8)$ & $10.3(5.2-17.0)$ & 0.553 & $8.9(5.8-21.9)$ & $12.2(7.7-16.7)$ & 0.715 \\
\hline Serum lactate - mmol/L (\%) & $3.8(3.0-4.9)$ & $3.9(2.8-5.5)$ & 0.820 & $4.7(3.7-6.3)$ & $3.6(2.8-6.3)$ & 0.091 \\
\hline \multicolumn{7}{|c|}{$\begin{array}{l}\text { Data presented as mean } \pm \text { SD or median (interquartile range), depending on distribution. } \\
\text { cell/cu.mm: Cell per cubic millimeter, IQR: Interquartile range, MAP: Mean arterial pressure, min: Minute, mmHg: Millimeter of mercury, NEWS: National Early Warning Score, }\end{array}$} \\
\hline
\end{tabular}

in sepsis (6). The findings from these studies led to the surviving sepsis campaign in 2018 the outcomes of which recommended the administration of adequate empirical intravenous antibiotics within the first hour of treatment. In addition, it was found that the activation of the sepsis care system in the ED causes faster intervention than conventional treatment (18-20). Furthermore, activation of the system was found to reduce door-to-lab time significantly. Such findings were in agreement with a study by Hayden et al. (8) which concluded that faster lab results resulted in a faster diagnosis, using the SOFA score, according to the Third International Consensus Definitions for Sepsis and Septic Shock (SEPSIS-3) definition. However, in our study there were no differences with regard to time to fluid resuscitation. This resulted from patients with a lower MAP score in our study being triaged 


\begin{tabular}{|c|c|c|c|c|c|c|}
\hline & \multicolumn{3}{|c|}{ Normotensive group } & \multicolumn{3}{|l|}{ Hypotensive group } \\
\hline & $\begin{array}{l}\text { Pre-intervention } \\
(\mathrm{n}=71)\end{array}$ & $\begin{array}{l}\text { Post-intervention } \\
(n=58)\end{array}$ & p value & $\begin{array}{l}\text { Pre-intervention } \\
(\mathrm{n}=47)\end{array}$ & $\begin{array}{l}\text { Post-intervention } \\
(n=44)\end{array}$ & $p$ value \\
\hline Door-to-antibiotic time - $\min (\mathrm{IQR})$ & $44.5(30.0-64.3)$ & $25.0(20.5-51.5)$ & 0.003 & $41.0(23.0-58.0)$ & $37.5(19.0-83.5)$ & 0.617 \\
\hline Door-to-IV bolus time - $\min (\mathrm{IQR})$ & - & - & - & $12.0(6.0-32.0)$ & $10.00(5.0-24.8)$ & 0.143 \\
\hline Door-to-lab time taken - min (IQR) & $16.5(6.8-27.3)$ & $11.50(5.5-20.0)$ & 0.005 & $9.0(3.0-15.0)$ & $9.0(5.0-18.3)$ & 0.299 \\
\hline Lactate clearance - \% (IQR) & $35.2(9.2-45.3)$ & $35.0(7.5-47.2)$ & 0.890 & $22.0(13.3-38.2)$ & $13.3(-1.3-31.4)$ & 0.335 \\
\hline Time to admission decision - $\min$ & $260.4 \pm 104.9$ & $229.8 \pm 108.1$ & 0.109 & $213.0(173.0-300.0)$ & $273.0(196.3-327.5)$ & 0.025 \\
\hline Average hospital LOS - day (IQR) & $8.0(6.0-21.5)$ & $14.5(8.3-17.8)$ & 0.863 & $9.0(4.0-32.0)$ & $7.0(5.0-12.0)$ & 0.633 \\
\hline ICU free days - day (IQR) & $7.0(4.0-13.0)$ & $7.0(4.0-10.5)$ & 0.435 & $4.0(0.0-9.0)$ & $6.0(2.0-9.0)$ & 0.296 \\
\hline Ventilator free days - day (IQR) & $7.0(4.0-12.3)$ & $7.0(3.0-10.5)$ & 0.642 & $4.0(0.0-9.0)$ & $6.0(2.0-9.0)$ & 0.279 \\
\hline 28-day mortality rate - n (\%) & $11(15.7)$ & $15(25.9)$ & 0.230 & $24(51.0)$ & $16(36.4)$ & 0.230 \\
\hline
\end{tabular}

as a resuscitation case, and thus most patients would receive rapid fluid administration regardless of sepsis fast track use. This study shows no statistical difference in the 28-day mortality rate, possibly due to insufficient numbers of patients, or possibly that the door-to-antibiotic time in the pre-intervention group was less than other studies (18). Future studies with larger number of patients are needed to verify this.

In our subgroup analysis, we found that the activation of sepsis fast track reduced door-to-antibiotic time and door-to-lab time. However, for the hypotensive group, there were no significant differences in door-to-antibiotic time, door-to-lab time, and time to fluid administration between the pre- and post-intervention groups. An explanation for this is probably because patients in the hypotensive group were triaged as resuscitation cases, thus would receive prompt intervention regardless of protocol activation. However, this study does highlight how the protocol helps personnel to have an increased focus on sepsis as regards normotensive patients. Time to admission decision was increased in the study, a finding which was in line with those published by both Hayden et al. (8) and Permpikul et al. (21). Both studies found that rapid diagnosis and management in the "golden hour", including administering norepinephrine in septic shock patients reduced the duration of shock, pulmonary edema, and new-onset arrhythmia, another field of septic shock which needs further investigation.

\section{Study Limitations}

As this study was a quasi-experimental study, some information obtained retrospectively may be missing. As for the postintervention group, using NEWS as a screening tool screens by the severity of the case; thus, this may not be inclusive of instances where the infection was not initially suspected. In addition, the numbers of patients with sepsis according to the SEPSIS-3 definition were not analyzed in this study as a diagnostic study; nonetheless, the ED uses the SEPSIS-3 definition to guide diagnosis (1). Additionally, if at any time the physician does not suspect sepsis after activation of the protocol, the physician will indicate in the records that sepsis was not suspected and re-designate the case as an infectious or non-infectious case instead.

\section{Conclusion}

Using NEWS as a trigger tool to activate the sepsis fast track protocol helps to reduce the door-to-antibiotic time in patients suspected of sepsis in the ED.

\section{Ethics}

Ethics Committee Approval: This study was a retrospective and prospective, quasi-experimental study which was approved by the Research Ethics Committee of Faculty of Medicine of Chiang Mai University (no: 377/2018, date: 03.10.2018).

Informed Consent: Written informed consent was obtained from patients who were enrolled on the prospective study.

Peer-review: Externally peer-reviewed.

\section{Authorship Contributions}

Surgical and Medical Practices: P.T., B.W., T.C., T.T., Concept: P.T., B.W., T.C., T.T., Design: P.T., B.W., T.C., T.T., Data Collection or Processing: P.T., B.W., T.C., T.T., Analysis or Interpretation: P.T., B.W., T.C., T.T., Literature Search: P.T., B.W., T.C., T.T., Writing: P.T., B.W., T.C., T.T.

Conflict of Interest: The authors declare they have no competing interest.

Financial Disclosure: Financial Disclosure: This study received Faculty of Medicine Research Fund of Chiang Mai University (grant no. 068-2562). 


\section{References}

1. Levy MM, Evans LE, Rhodes A. The Surviving Sepsis Campaign Bundle: 2018 update. Intensive Care Med. 2018;44:925-8.

2. Rhee C, Dantes R, Epstein L, Murphy DJ, Seymour CW, Iwashyna TJ, et al. Incidence and Trends of Sepsis in US Hospitals Using Clinical vs Claims Data, 2009-2014. JAMA. 2017;318:1241-9.

3. Ministry of Public Health of Thailand. Mortality rate in patients with community-acquired severe sepsis or septic shock [Internet]. Bangkok: Ministry of Public Health of Thailand; 2018 [cited 2019 Oct 1]. Available from: http://healthkpi.moph.go.th/kpi/kpi-list/view/?id=964

4. Hatfield KM, Dantes RB, Baggs J, Sapiano MRP, Fiore AE, Jernigan JA, et al. Assessing Variability in Hospital-Level Mortality Among U.S. Medicare Beneficiaries with Hospitalizations for Severe Sepsis and Septic Shock. Crit Care Med. 2018;46:1753-60.

5. de Groot B, Ansems A, Gerling DH, Rijpsma D, van Amstel P, Linzel D, et al. The association between time to antibiotics and relevant clinical outcomes in emergency department patients with various stages of sepsis: a prospective multi-center study. Crit Care. 2015;19:194.

6. Seymour CW, Gesten F, Prescott HC, Friedrich ME, Iwashyna TJ, Phillips GS, et al. Time to Treatment and Mortality during Mandated Emergency Care for Sepsis. N Engl J Med. 2017;376:2235-44.

7. Zasowski EJ, Claeys KC, Lagnf AM, Davis SL, Rybak MJ. Time Is of the Essence: The Impact of Delayed Antibiotic Therapy on Patient Outcomes in HospitalOnset Enterococcal Bloodstream Infections. Clin Infect Dis. 2016;62:1242-50.

8. Hayden GE, Tuuri RE, Scott R, Losek JD, Blackshaw AM, Schoenling AJ, et al. Triage sepsis alert and sepsis protocol lower times to fluids and antibiotics in the ED. Am J Emerg Med. 2016;34:1-9.

9. Amland RC, Sutariya BB. Quick Sequential [Sepsis-Related] Organ Failure Assessment (qSOFA) and St. John Sepsis Surveillance Agent to Detect Patients at Risk of Sepsis: An Observational Cohort Study. Am J Med Qual. 2018;33:507.

10. Askim A, Moser F, Gustad LT, Stene H, Gundersen M, Asvold BO, et al. Poor performance of quick-SOFA (qSOFA) score in predicting severe sepsis and mortality - a prospective study of patients admitted with infection to the emergency department. Scand J Trauma Resusc Emerg Med. 2017;25:56.

11. Finkelsztein EJ, Jones DS, Ma KC, Pabon MA, Delgado T, Nakahira K, et al. Comparison of qSOFA and SIRS for predicting adverse outcomes of patients with suspicion of sepsis outside the intensive care unit. Crit Care. 2017;21:73.
12. Goulden R, Hoyle MC, Monis J, Railton D, Riley V, Martin P, et al. qSOFA, SIRS and NEWS for predicting inhospital mortality and ICU admission in emergency admissions treated as sepsis. Emerg Med J. 2018;35:345-9.

13. Smith GB, Prytherch DR, Meredith P, Schmidt PE, Featherstone PI. The ability of the National Early Warning Score (NEWS) to discriminate patients at risk of early cardiac arrest, unanticipated intensive care unit admission, and death. Resuscitation. 2013;84:465-70.

14. Churpek MM, Snyder A, Han X, Sokol S, Pettit N, Howell MD, et al. Quick Sepsis-related Organ Failure Assessment, Systemic Inflammatory Response Syndrome, and Early Warning Scores for Detecting Clinical Deterioration in Infected Patients outside the Intensive Care Unit. Am J Respir Crit Care Med. 2017;195:906-11.

15. Rosner B. Fundamentals of Biostatistics, 8th ed. Boston, MA: Cengage Learning; 2016. http://galaxy.ustc.edu.cn:30803/zhangwen/Biostatistics/ Fundamentals+of+Biostatistics+8th+edition.pdf

16. Usman OA, Usman AA, Ward MA. Comparison of SIRS, qSOFA, and NEWS for the early identification of sepsis in the Emergency Department. Am J Emerg Med. 2019;37:1490-7.

17. Thodphetch M, Chenthanakij B, Wittayachamnankul B, Tangsuwanaruk T. Comparison of modified Systemic Inflammatory Response Syndrome (mSIRS), quick Sequential Organ Failure Assessment (qSOFA), modified National Early Warning Score (mNEWS) and modified Search Out Severity (mSOS) score for Predicting in-hospital Mortality among Patients with Suspected Infection Presenting to the Emergency Department. Thai J Emerg Med. 2019;1:120-1.

18. Robert Boter N, Modol Deltell JM, Casas Garcia I, Rocamora Blanch G, Llados Beltran G, Carreres Molas A. Activation of a code sepsis in the emergency department is associated with a decrease in mortality. Med Clin (Barc). 2019; 152:255-60.

19. Ferreras Amez JM, Arribas Entrala B, Sarrat Torres MA, Garcia Noain A, Caudevilla Martinez A, Colas Oros C, et al. Before-after study of the effect of implementing a sepsis code for emergency departments in the community of Aragon. Emergencias. 2017;29:154-60.

20. Bruce HR, Maiden J, Fedullo PF, Kim SC. Impact of nurse-initiated ED sepsis protocol on compliance with sepsis bundles, time to initial antibiotic administration, and in-hospital mortality. J Emerg Nurs. 2015;41:130-7.

21. Permpikul C, Tongyoo S, Viarasilpa T, Trainarongsakul T, Chakorn T, Udompanturak S. Early Use of Norepinephrine in Septic Shock Resuscitation (CENSER). A Randomized Trial. Am J Respir Crit Care Med. 2019;199:1097-105. 